\title{
Technè
}

La science au service de l'histoire de l'art et de la préservation des biens culturels

$40 \mid 2014$

Thérapéia. Polychromie et restauration de la sculpture dans l'Antiquité

\section{Deux portraits chypriotes en calcaire polychrome}

Two Cypriot portraits in polychrome limestone

Nicolas Bel, Christine Pariselle, Sandrine Pagès-Camagna, Yvan Coquinot et Noëlle Timbart

\section{(2) OpenEdition}

\section{Journals}

\section{Édition électronique}

URL : https://journals.openedition.org/techne/3815

DOI : $10.4000 /$ techne.3815

ISSN : 2534-5168

Éditeur

C2RMF

\section{Édition imprimée}

Date de publication : 26 novembre 2014

Pagination : 96-105

ISBN : 978-2-7118-6218-4

ISSN : $1254-7867$

Référence électronique

Nicolas Bel, Christine Pariselle, Sandrine Pagès-Camagna, Yvan Coquinot et Noëlle Timbart, « Deux portraits chypriotes en calcaire polychrome », Technè [En ligne], 40 | 2014, mis en ligne le 24 juillet 2020, consulté le 08 février 2022. URL : http://journals.openedition.org/techne/3815 ; DOI : https:// doi.org/10.4000/techne.3815

\section{(c) (†) $\ominus$}

La revue Technè. La science au service de l'histoire de l'art et de la préservation des biens culturels est mise à disposition selon les termes de la Licence Creative Commons Attribution - Pas d'Utilisation Commerciale - Pas de Modification 4.0 International. 


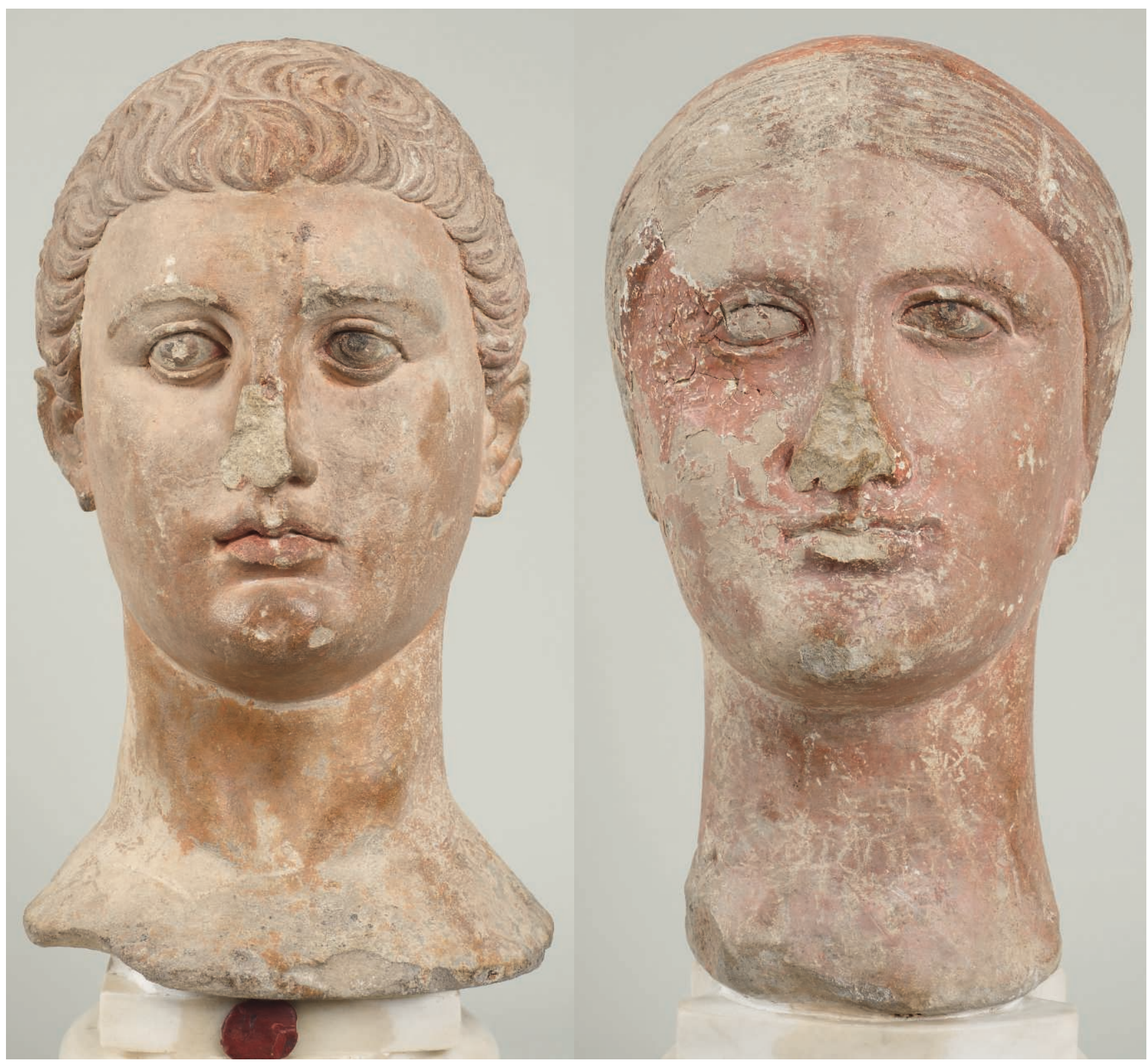

Fig. 1. Tête masculine

( $\mathrm{N}$ 1477), vue générale (face), H. 25,5 cm.

(c) C2RMF/Anne Chauvet.
Fig. 2. Tête féminine

(N 1086), vue générale (face), H. 25,5 cm. (c) C2RMF/Anne Chauvet. 
Nicolas Bel

Christine Pariselle

Sandrine Pagès-Camagna

Yvan Coquinot

Avec la collaboration

de Noëlle Timbart

\section{Deux portraits chypriotes en calcaire polychrome}

Two Cypriot portraits in polychrome limestone

Résumé. Le projet de restauration de deux têtes chypriotes conservées au département des Antiquités orientales du Louvre a été préparé par un inventaire diagnostique des altérations, étude qui, alliée à celle des polychromies, a permis de séquencer l'histoire matérielle des deux auvres aux destinées semble-t-il communes et exceptionnelles. À partir de la reconnaissance de deux étapes picturales anciennes, impliquant une restauration antique ou médiévale, la succession des dégradations tant superficielles que structurelles a pu être proposée pour chacune des deux sculptures. Les caractéristiques pétrographiques et la composition chimique élémentaire des pierres les constituant ont été mises en évidence, ce qui a permis une révision des résultats des analyses réalisées en 1987 par le laboratoire du Centre pour les Sciences Archéologiques de l'université de Georgia.

Mots-clés. Chypre, sculpture, calcaire, polychromie, période romaine, restauration antique, réutilisation.

Les deux têtes sculptées étudiées ici (fig. 1 et 2) ${ }^{1}$ appartiennent au fonds Guillaume-Rey du département des Antiquités orientales (musée du Louvre) ${ }^{2}$. Emmanuel Guillaume-Rey (18371916), «G. Rey» dans les inventaires du Louvre, a bénéficié de deux missions d'État successives en Orient (1857-1858, 1864-1865) ${ }^{3}$, au cours desquelles il a fait escale à Chypre, ce qui lui a permis de sillonner les sites antiques de l'île, en compagnie de l'archéologue Eugène Grasset, et aussi, sans doute, de fréquenter les marchands de Larnaca. Les nombreuses antiquités qu'il en rapporta, et qui furent dévolues, selon l'usage, principalement au Louvre, vinrent alimenter les toutes jeunes collections chypriotes du musée ${ }^{4}$, qui furent ensuite grossies de celles rapportées par Saulcy, Luynes, Voguë ou les frères Duthoit. Ces arrivées massives ne laissèrent pas le loisir au conservateur, A. de Longperrier, de collecter toutes les informations propres à éclairer les contextes d'acquisition des œuvres. Il fallut attendre le travail de Léon Heuzey, puis celui d'Annie Caubet pour reconstituer des listes précises, sans pouvoir toujours identifier les lieux de trouvaille.

\begin{abstract}
Prior to the restoration of two Cypriot heads now in the Louvre's Department of Near Eastern Antiquities, a diagnostic inventory of the alterations previously made to them was drawn up. This study, together with another on their polychromy, enabled us to establish the material history of two works whose destinies seem to have been both shared and exceptional. Having identified two early pictorial stages, implying either an ancient or medieval restoration, the sequence of both their superficial and structural deterioration was proposed for each of the two sculptures. The petrographic characteristics and elementary chemical composition of the stones in which they were carved were brought to light, which enabled us to revise the results of the analyses made in 1987 in the laboratory of the Center for Archaeological Sciences at the University of Georgia.

Keywords. Cyprus, sculpture, limestone, polychromy, Roman period, ancient restoration, re-use.
\end{abstract}

Constitué de figurines en terre cuite (Âge du Fer, époque hellénistique) et de sculptures en calcaire (représentations de dédicants, têtes de divinités), le fonds G. Rey inclut également deux têtes sculptées polychromes un peu isolées par leur style, qui semblent se rattacher aux séries typologiques habituellement présentes à Chypre, tout en se référant au type du portrait romain. La tête masculine (N 1477), de taille naturelle, montre un jeune homme imberbe à la chevelure ondulée en petites mèches, au visage arrondi; le départ des épaules est représenté (fig. 1). Les carnations varient du rosé à l'orangé. Les cheveux sont peints en brun-rouge. La bouche est rehaussée de rouge. Le traitement pictural des yeux est détaillé : les pupilles sont peintes en brun, l'iris en noir, du blanc bleuté colore le fond de l'œil, l'intérieur des paupières supérieure et inférieure est souligné de brun, du rouge a été utilisé pour marquer la caroncule lacrymale ou chantus. Un trait de peinture brune colore l'arc des sourcils. La tête féminine (N 1086), de taille presque naturelle, a les cheveux coiffés en chignon et pris dans un foulard; le visage est allongé, les

Nicolas Bel, conservateur du patrimoine, DRAC Limousin (nicolas.bel@culture.gouv.fr). Christine Pariselle, restauratrice indépendante (christinepariselle@free.fr). Sandrine Pagès-Camagna, ingénieur de recherche, département Recherche, C2RMF (sandrine.pages@culture. gouv.fr). Yvan Coquinot, ingénieur de recherche, département Recherche, C2RMF (yvan.coquinot@culture.gouv.fr). Avec la collaboration de Noëlle Timbart, conservateur du patrimoine chargé des antiquités égyptiennes et orientales, département Restauration, C2RMF (noelle.timbart@culture.gouv.fr). 
yeux tombants; les reliefs sont peu marqués (fig. 2). Les carnations sont d'un rose assez soutenu. Les cheveux sont peints en brun-rouge, le foulard en orange. La bouche est rehaussée de rouge. Le traitement pictural des yeux est similaire à celui du personnage masculin (code identique pour le tracé et les couleurs), mais son exécution semble plus hâtive ou moins soignée. La ligne des sourcils était colorée de brun ou de noir (traces seules conservées). Les disques formant les pendentifs d'oreilles sont teintés de rouge. Dans les deux cas, les traits du visage sont beaucoup plus individualisés que sur les têtes sculptées traditionnellement déposées en ex-voto dans les sanctuaires chypriotes (Amathonte, Pyla, Idalion, Golgoi) dès le VI ${ }^{\mathrm{e}}$ siècle av. J.-C. ${ }^{5}$ En outre, l'observation de certains détails (trous à la base du cou, préparation du lit d'attente) corps, et amène à s'interroger sur l'usage - ou les usages - qui ont pu en être faits. Aux questions de style et d'usage s'en ajoute une troisième: la polychromie encore très présente sur les deux têtes montre à l'œil nu des superpositions et des décalages difficiles à expliquer, tandis que des anomalies structurelles sont manifestes: ces objets ont-ils pu être repeints, restaurés au cours de leur(s) utilisation(s) antique(s)?

La préparation d'un nouveau parcours muséographique, consacré à l'Orient romain ${ }^{6}$, fut l'occasion de se pencher sur ces questions, dans la mesure où les deux pièces devaient faire l'objet d'une intervention de restauration en vue de leur présentation au public. Le nettoyage et la consolidation ponctuelle de la polychromie s'accompagnèrent donc d'une caractérisation de la pierre, ainsi que d'une étude approfondie de cette polychromie, pour tenter d'en savoir davantage sur la chronologie des usages de ces deux ouvres.

\section{Apport de l'étude pétrographique}

Seules quelques informations disparates quant à la nature de la pierre dans laquelle ont été sculptées ces têtes étaient connues jusque-là. Dans son catalogue, Antoine Hermary précisait que le matériau utilisé pour la fabrication de la tête

féminine était un calcaire compact. En 1984, Annie Caubet indiquait que la tête était en calcaire ${ }^{7}$. Or, en 1987, les investigations effectuées par le laboratoire de l'université de Georgia avaient révélé que la pierre constitutive de la tête féminine retenue pour la sélection de dix sculptures sélectionnées dans le cadre d'un programme d'analyses était du marbre pouvant provenir de Turquie, des carrières d'Aphrodisias ou d'Éphèse ${ }^{8}$. Antoine Hermary n'indiquait pas la nature de la pierre constitutive de la tête masculine, mais Annie Caubet précisait qu'il s'agissait de calcaire ${ }^{9}$.

L'occasion était donc donnée d'apporter un nouvel éclairage grâce à une étude pétrographique ${ }^{10}$. Un prélèvement de pierre de quelques millimètres a été effectué sur chacune des têtes sculptées, à la base du cou. Les fragments de roches ont ensuite été inclus dans une résine Epoxy afin de réaliser des lames minces polies destinées aux observations pétrographiques en microscopie optique. Par ailleurs, la composition chimique des deux pierres a été déterminée en PIXE, à l'aide de l'Accélérateur Grand Louvre d'Analyse Élémentaire (AGLAE).

Les compositions des deux pierres et leurs caractéristiques pétrographiques sont identiques (fig. 3). Il s'agit de calcaires provenant de la même formation géologique.

Macroscopiquement, la pierre des deux sculptures correspond à un calcaire fin de couleur beige, riche en microfossiles, visibles à la loupe. Ceux-ci lui confèrent un aspect finement "grumeleux». Au microscope optique, le calcaire présente une matrice micritique qui occupe entre 50 et $70 \%$ du volume de la roche. Les éléments figurés (= allochèmes) correspondent principalement à des foraminifères planctoniques (plus de $95 \%$ des éléments figurés présents) dont la plupart des loges sont vides. D'après la classification de Folk (1959), ce calcaire est une biomicrite. Les foraminifères sont exclusivement des Globigérines (g/ Globigerina). L'âge de ce calcaire se situe entre le Cénomanien (Crétacé supérieur) et le Miocène supérieur (Néogène inférieur à moyen).

Ce calcaire présente un microfaciès relativement fréquent dans les formations calcaires de milieu pélagique du Crétacé supérieur, du Paléogène et du Néogène. Des calcaires de ce

\begin{tabular}{|c|c|c|c|c|c|c|c|c|c|c|c|c|c|c|c|c|c|}
\hline \multirow[t]{2}{*}{ Ref. Ech. } & \multicolumn{11}{|c|}{ Composition (\% massique d'oxydes) } & \multicolumn{5}{|l|}{ ppm } & \multirow{3}{*}{$\begin{array}{l}\text { Fig. 3. Compositions } \\
\text { élémentaires des } \\
\text { calcaires des deux têtes } \\
\text { sculptées et du standard } \\
\text { déterminées par PIXE } \\
\text { à l'aide d'AGLAE. }\end{array}$} \\
\hline & $\mathrm{Na}_{2} \mathrm{O}$ & $\mathrm{MgO}$ & $\mathrm{Al}_{2} \mathrm{O}_{3}$ & $\mathrm{SiO}_{2}$ & $\mathrm{SO}_{3}$ & $\mathrm{Cl}$ & $\mathrm{K}_{2} \mathrm{O}$ & $\mathrm{CaO}$ & $\mathrm{TiO}_{2}$ & $\mathrm{MnO}$ & $\mathrm{Fe}_{2} \mathrm{O}_{3}$ & $\mathrm{Ni}$ & $\mathrm{Cu}$ & $\mathrm{Zn}$ & $\mathrm{Sr}$ & Total (\%) & \\
\hline N1086 & 0.20 & 0.75 & 1.34 & 4.60 & 1.16 & 0.69 & - & 89.98 & 0.10 & 0.05 & 0.83 & 19 & 44 & 43 & 1250 & 99.8 & \\
\hline N1477 & 0.13 & 0.82 & 1.51 & 5.79 & 0.93 & 0.62 & - & 88.85 & 0.12 & 0.05 & 1.10 & $\begin{array}{c}16 \\
\text { ppm }\end{array}$ & 45 & 32 & 1260 & 100 & $\begin{array}{l}\text { Composition exprimée } \\
\text { en pourcentage } \\
\text { massique d'oxydes et } \\
\text { en ppm (partie par }\end{array}$ \\
\hline DRNi1 & 2.68 & 3.87 & 17.48 & 55.08 & 0.44 & 0.05 & 1.63 & 6.87 & 1.14 & 0.21 & 10.29 & 19 & 25 & 133 & 437 & 99.8 & $\begin{array}{l}\text { million). Valeurs en } \\
\text { rouge: au niveau de la }\end{array}$ \\
\hline DRNi2 & 2.75 & 3.96 & 17.95 & 55.01 & 0.46 & 0.04 & 1.65 & 6.89 & 1.03 & 0.21 & 9.77 & 26 & 25 & 160 & 465 & 99.8 & $\begin{array}{l}\text { limite de détection. } \\
\text { (c) Yvan Coquinot. }\end{array}$ \\
\hline & $\mathrm{Na}_{2} \mathrm{O}$ & $\mathrm{MgO}$ & $\mathrm{Al}_{2} \mathrm{O}_{3}$ & $\mathrm{SiO}_{2}$ & & & $\mathrm{~K}_{2} \mathrm{O}$ & $\mathrm{CaO}$ & $\mathrm{TiO}_{2}$ & $\mathrm{MnO}$ & $\mathrm{Fe}_{2} \mathrm{O}_{3}$ & $\mathrm{Ni}$ & $\mathrm{Cu}$ & $\mathrm{Zn}$ & $\mathrm{Sr}$ & & * DRN (ICP-AES et ICI \\
\hline $\mathrm{DRN}^{*}$ & 2.99 & 4.4 & 17.52 & 52.85 & & & 1.7 & 7.05 & 1.09 & 0.22 & 9.7 & 15 & 50 & 145 & 400 & & MS) \\
\hline
\end{tabular}




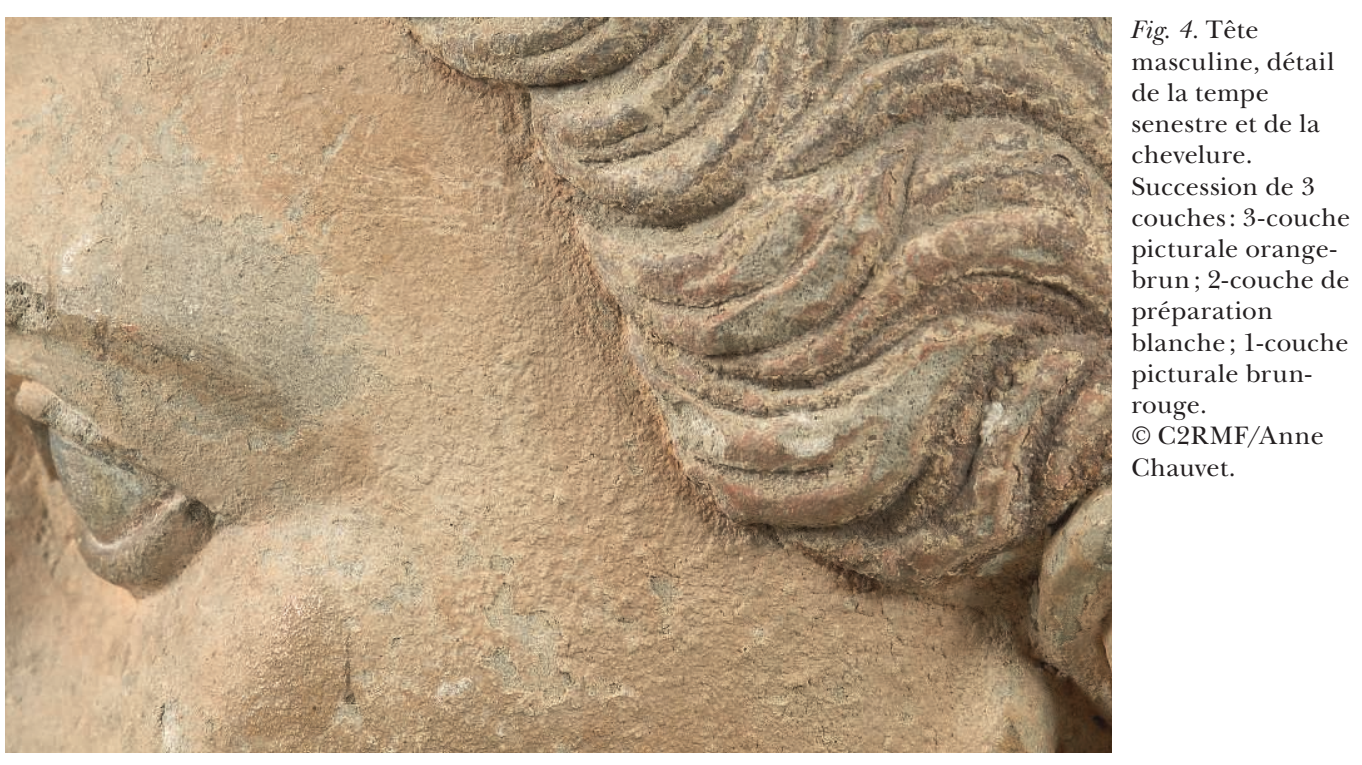

Fig. 5. Tête

féminine, côté dextre du visage restauré, éclats profonds bouchés à l'aide d'un matériau blanc craquelé, ensemble des surfaces repeints. (C) C2RMF/Anne Chauvet.

type affleurent ainsi en Égypte, en Syrie, en Italie, en Turquie, sur Chypre et sans doute dans d'autres pays bordant la Méditerranée. Un calcaire similaire à celui des deux têtes sculptées est présent dans la formation Miocène de Pakhna, dans le sud de Chypre, au niveau des bassins de Khalassa et de Maroni (Eaton et Roberston, 1993). Toutefois, il n'est pas certain que le calcaire des deux sculptures provienne de cette formation.

\section{Description des états de surface actuels}

Au premier examen des deux têtes, en plus d'un encrassement important (dont le nettoyage a constitué l'essentiel de la restauration de 2009), des anomalies structurelles et picturales ont immédiatement été détectées ${ }^{11}$.

L'arrière de la tête masculine a été manifestement retaillé. Cinq coups de ciseau ont permis l'affranchissement d'un fragment circulaire de taille et d'épaisseur importantes, et l'arrière du crâne a ainsi été tronqué de manière régulière. On remarque qu'après cette intervention, la chevelure a été entièrement repeinte en brun-rouge (couche continue passant sur les traces de ciseau et le plan de fracture et recouvrant le reste de la chevelure) (fig. 4).

Le côté droit de la tête féminine a été restauré. Trois éclats, apparemment très étendus et profonds, affectant le côté droit du visage (tempe et bord externe de l'œil) et du foulard (au niveau du dessus et de l'arrière de l'oreille), ont été bouchés à l'aide d'un matériau blanc. L'ensemble des surfaces a été repeint au moment de cette intervention structurelle: on observe une continuité des couches picturales sur les parties gauche et droite de la tête (carnation, yeux, foulard). L'analyse de la partie droite de la tête est particulièrement significative. Les éclats ont été bouchés avec un produit qui s'est manifestement craquelé en cours de restauration. Dans les creux des fissures, on observe de la peinture rose et des sédiments, ce qui induit que l'altération du produit de

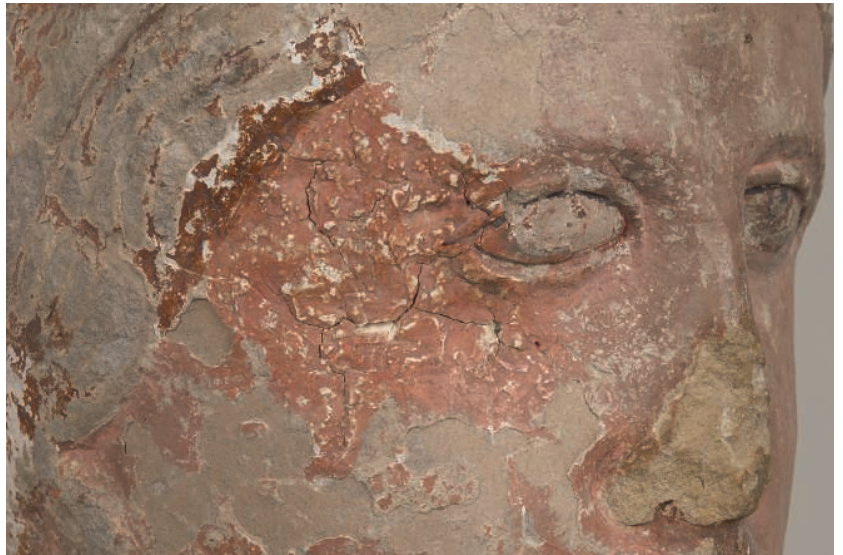

bouchage est survenue en cours de restauration, pendant son séchage (retrait lié à sa composition ou matériau non adapté à un bouchage profond). L'ensemble de la tête a été repeint (continuité des couches picturales sur les parties gauche et droite de la tête). Les différences de tonalités des couches picturales perçues sur le visage et le foulard peuvent s'expliquer par les différences de porosité des surfaces restaurées et intègres juxtaposées (fig. 5).

Sur les deux têtes, un indice constitue un témoignage contextuel et chronologique significatif. Sur les surfaces des visages et des cous, ainsi que sur le foulard de la représentation féminine, les stigmates laissés par des empreintes de racines ont été observés. Le fond des empreintes est coloré en rose ou orange, selon leur localisation (carnations ou foulard), ce qui confirme l'exécution d'une reprise du traitement pictural après l'impression de ces racines. Par ailleurs, la présence de sédiments ancrés sur les fonds des sillons colorés indique que les repeints sont anciens (antérieurs à l'enfouissement).

Au regard de ces anomalies structurelles et témoignages superficiels, on pouvait déjà supposer que les deux sculptures avaient fait l'objet d'un remploi. Pour une deuxième utilisation, la tête masculine a été affranchie et la tête féminine 

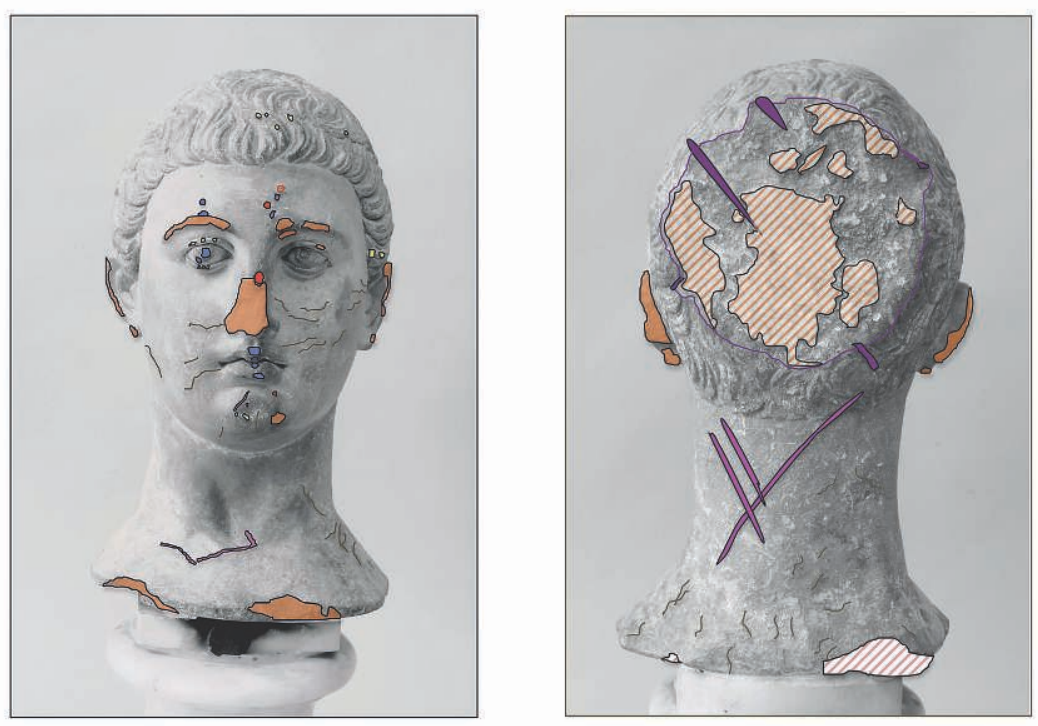

Fig. 6. Tête masculine, relevé des altérations. (c) Christine Pariselle.
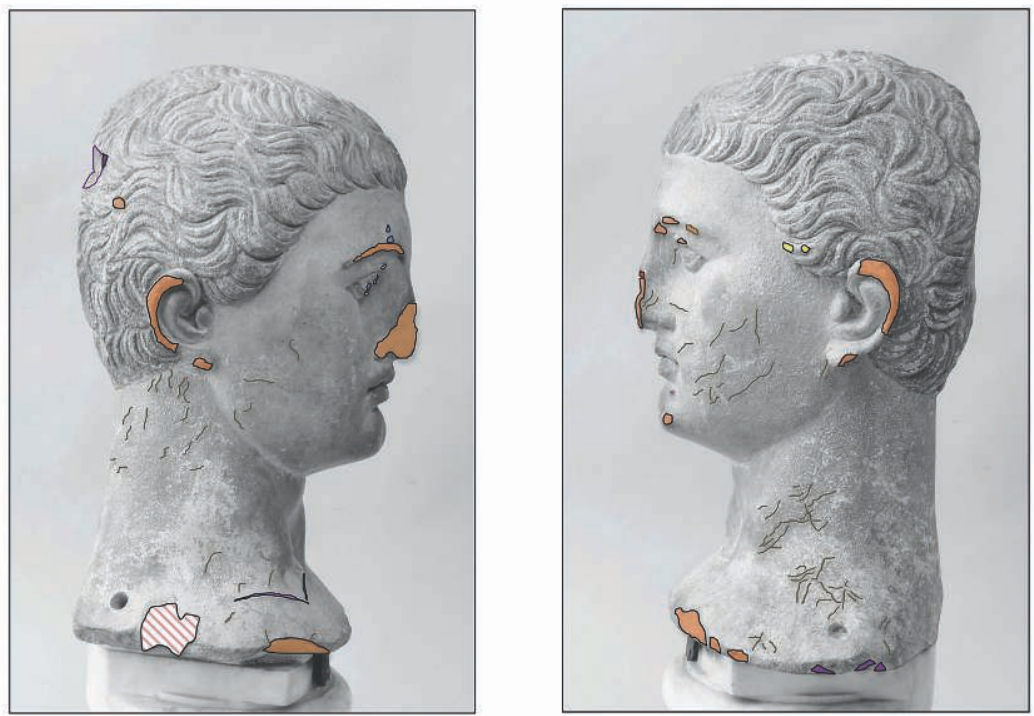

- Cavités / Inclusions minérales rouges

- Pertes liées à la présence d'inclusions minérales

- Empreintes de fines racines ou de lianes (antérieures à la polychromie secondaire)

- Eraflures antérieures à la polychromie secondaire

- Cassures antérieures à la polychromie secondaire

- Coups de ciseau (antérieurs à la polychromie secondaire)

- Cassures et éclats postérieurs à la polychromie secondaire

Eclats récents

restaurée. Les deux œuvres ont été repeintes. Ces interventions ont été exécutées avant leur enfouissement, à la période antique ou médiévale.

L'examen approfondi des surfaces a permis d'établir un inventaire diagnostique des altérations. Les principales dégradations structurelles en relation avec leurs états de surface sont présentées sur les figures 6 et 7 :

- les altérations constitutives liées à la nature et la composition de la pierre. Sur le front, le nez et le menton de la tête masculine, on constate la présence de petits alvéoles apparemment liés à celle de nodules minéraux ou à leur départ (inclusions minérales noires et rouges ou de grains blancs affleurant par endroits sur le visage). Les parois de certaines cavités sont colorées de rose. -les altérations structurelles, accidentelles ou intentionnelles, cassures dont les surfaces apparaissent blanches, exemptes de traitement pictural. Elles sont antérieures à l'enfouissement (présence de sédiments sur les plans de fracture) et entrent dans la catégorie des cassures les plus récentes.

Dans le cas de la tête masculine, les éclats ou cassures plus profondes concernent: le nez (l'aile gauche est seulement préservée), les contours saillants et les lobes des deux oreilles ainsi que les arcs des sourcils. La partie plate de l'arrière de la tête est affectée par des impacts étendus, plus ou moins profonds, paraissant contemporains des cassures relevées sur le visage. Plusieurs bris sont également observés sur la face principale de la base de la sculpture. 

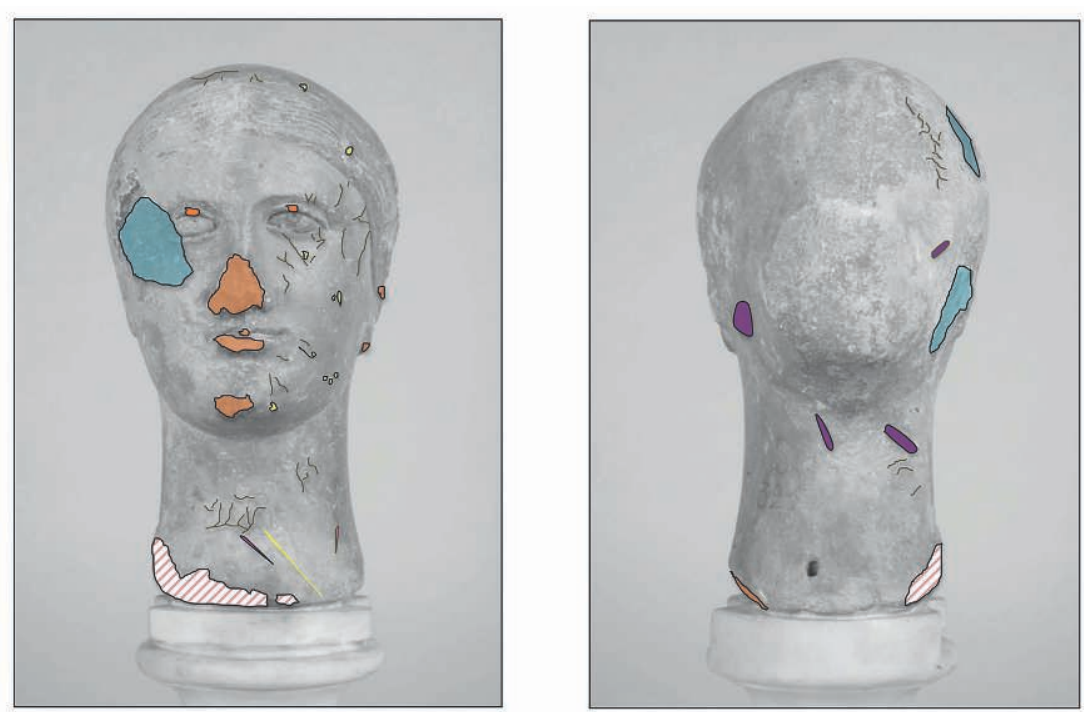

Fig. 7. Tête féminine, relevé des altérations. () Christine Pariselle.
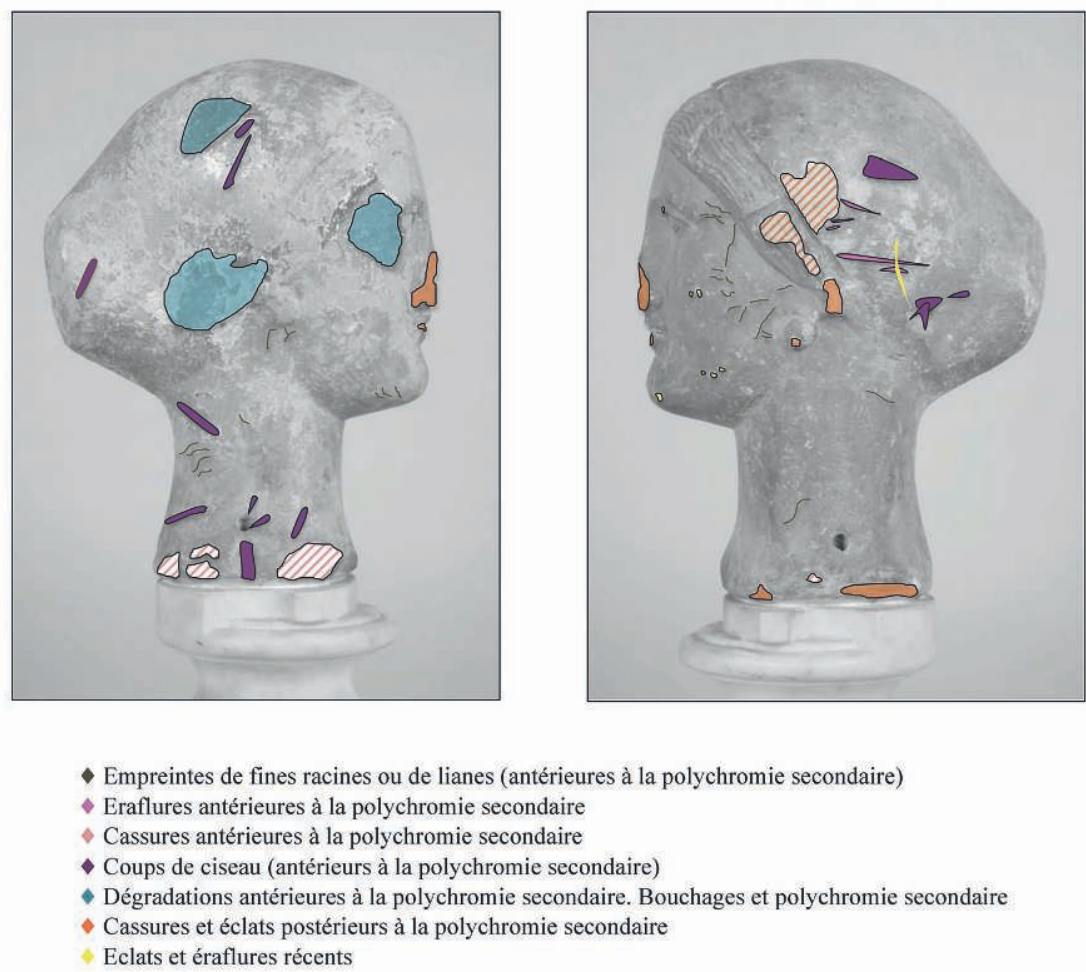

Dans le cas de la tête féminine, les cassures et les éclats les plus récents se trouvent essentiellement sur le visage. À l'instar de la tête masculine, le nez est cassé et seule l'aile gauche est conservée. On observe des éclats plus ou moins étendus sur les lèvres et le menton. Deux petites lacunes affectent les paupières supérieures. Le côté gauche de la sculpture est particulièrement altéré: lacunes sur l'oreille et sur le pendentif, pertes de matière étendues sur les cheveux et le foulard, cassures à la base du cou sont constatées.

Certaines de ces altérations superficielles ou structurelles témoignent d'une restauration ancienne, les surfaces étant recouvertes d'une couche de polychromie. Elles présentent un intérêt crucial pour la compréhension de l'histoire des œuvres (éraflures, cassures, coups de ciseau).
Dans le cas de la tête masculine, on relève en particulier une éraflure significative, longue et oblique, partant de la nuque et rejoignant la base de la chevelure. Cette éraflure est recouverte de la peinture brun-rouge colorant la chevelure. Par ailleurs, à la base de la tête, au revers et sur le côté droit, des cassures probablement accidentelles sont observées. Leurs surfaces sont roses.

Dans le cas de la tête féminine, sur son côté gauche et sur le foulard, on observe des éraflures groupées et orientées horizontalement. Leurs surfaces sont colorées de peinture orange.

À la base du cou, sur la face et le côté droit de la sculpture, plusieurs cassures, probablement accidentelles, sont visibles. Leurs surfaces sont roses. 
De nombreux coups de ciseau sont observés au revers et sur les côtés droit et gauche de l'œuvre.

Sur le côté droit:

- cinq coups de ciseau autour et à proximité de la mortaise, à la base de la tête;

- un coup de ciseau oblique, sur la nuque;

- un coup de ciseau oblique sur le chignon;

- deux coups de ciseau convergeant vers la lacune située sur le haut de la tête;

- un coup, peut-être, sur la lèvre inférieure.

Sur le côté gauche, sur le foulard: cinq coups de ciseau ou entailles.

Sur la nuque, au niveau du chignon: deux coups de ciseau obliques.

Les coups de ciseau portés autour de la mortaise située à la base de la tête sur son côté droit sont sans doute à l'origine de la dissociation de la tête d'avec son support/corps. Ceux repérés sur le haut de la tête sont vraisemblablement en relation avec la dégradation survenue sur le côté droit du visage: trois éclats profonds.

- les altérations contextuelles liées aux conditions de conservation (contextes d'utilisation, contexte d'enfouissement, contextes de conservation modernes) dont les stigmates laissés par des racines, colorés de rose scellé par la présence de sédiments. Si ce type de traces peut se former par contact avec des racines dans le contexte d'enfouissement, dans le cas des deux têtes étudiées, il semble que ces empreintes se sont créées en extérieur (abandon des œuvres dans un contexte végétal).

De manière générale, si la polychromie de la tête masculine est bien conservée, on note des lacunes des couches picturales dues à un écaillage localisé ou étendu de la couche préparatoire. Sur le côté gauche du visage, sur la tempe, la surface présente un aspect grumeleux sans doute lié à l'étape de fabrication de la préparation (broyage et mélange des constituants insuffisants?) ou à l'adhérence médiocre de la préparation au support (fig. 4). Sur le côté droit du cou et sur le

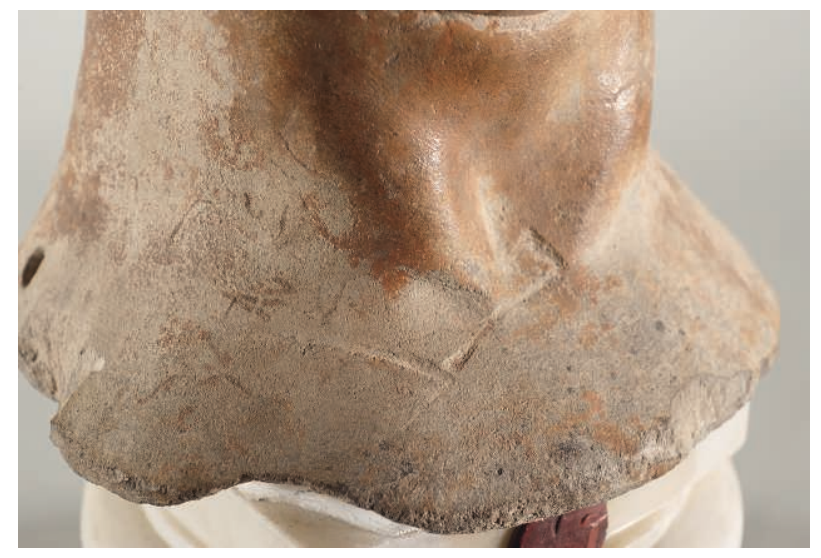

Fig. 8. Tête masculine, face, côté droit et base du cou, couche picturale ocre orangée lacunaire. (c) C2RMF/Anne Chauvet.

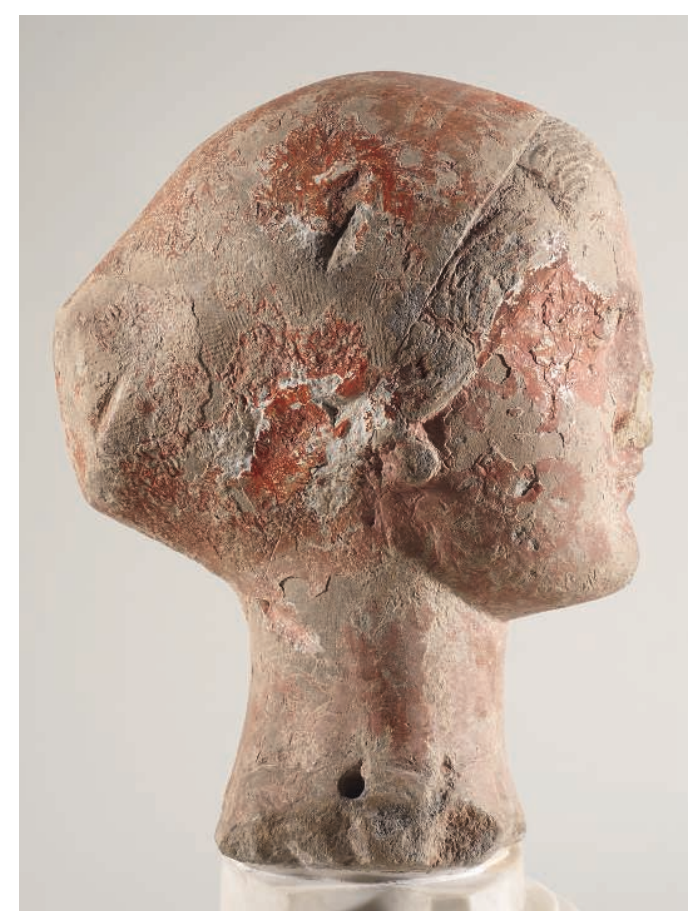

Fig. 9. Tête féminine, côté dextre, vue sous lumière rasante, traitement pictural fortement dégradé (c) $\mathrm{C} 2 \mathrm{RMF} /$ Anne Chauvet.

départ des épaules, la couche picturale ocre orangé est lacunaire (écaillage) (fig. 8). Dans le cas de la tête féminine, tandis que le traitement pictural est relativement bien préservé sur le côté gauche de la tête, sur le côté droit, il est extrêmement dégradé (disparition locale de la préparation et/ou des couches picturales). Il est probable que, dans le sol d'enfouissement, la tête ait reposé renversée sur ce côté (fig. 9).

Dans les deux cas, les vestiges de la polychromie initiale semblent ténus. Sur les cheveux de la tête féminine, une couche noire a été détectée, sur l'œil droit le tracé à la peinture brune de la pupille, laquelle apparaît plus petite que pour le tracé actuel, est visible. Sur les cheveux de la tête masculine, une couche orange est présente sous la couche brun-rouge.

\section{Étude des polychromies}

Afin de mieux appréhender les étapes du traitement coloré, une étude de polychromie ${ }^{12}$ a été menée, étayée par un dossier d'imagerie scientifique. Cette étude matérielle du traitement polychrome s'est fait durant la restauration des portraits dans les ateliers de restauration. Les éclairages sous ultraviolet n’ont pas révélé de matière organique particulière, mais ils ont permis d'apporter une meilleure lisibilité des empreintes végétales et des différents niveaux de polychromie, notamment sur les yeux. En outre, des micro-prélèvements ont été réalisés à l'aide d'un scalpel: quatre pour la tête masculine et cinq pour la tête féminine ${ }^{13}$. L'étude de la polychromie des têtes s'est donc limitée à cet échantillonnage, localisé sur des zones pouvant comporter à la fois des niveaux profonds, ainsi que des stratigraphies plus complexes. Les échantillons les plus grands ont été inclus en résine afin de réaliser une coupe stratigraphique. Les plus petits ont été analysés directement. 


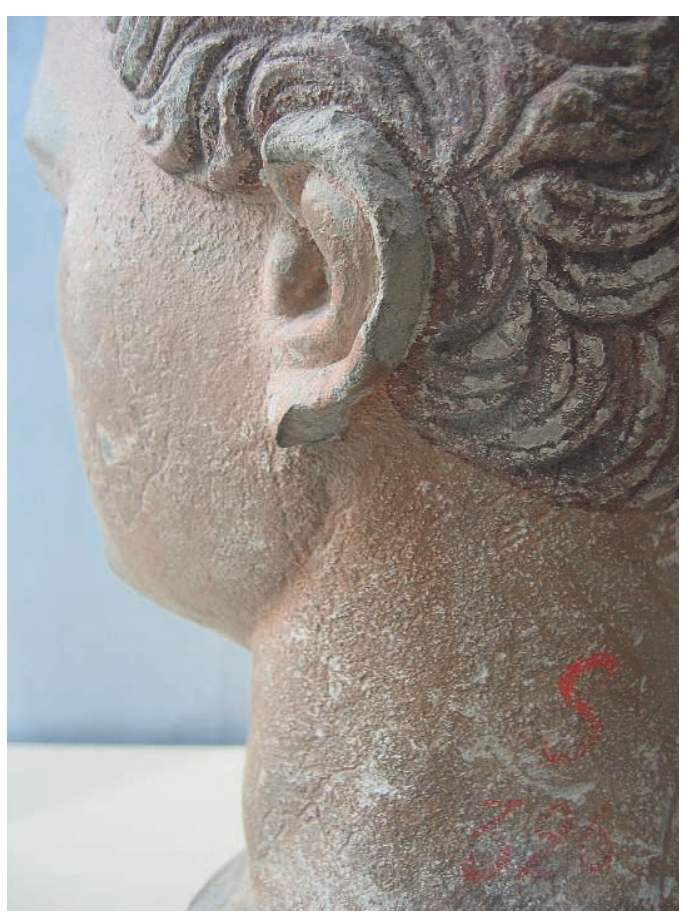

L'observation au microscope optique, dans le visible et sous lumière ultraviolette, et l'examen en microscopie électronique à balayage ont été complétés par l'analyse élémentaire de rayons X par séparation dispersive en énergie associée au MEB. Afin d'affiner l'identification des composés minéraux, la microspectométrie Raman a été réalisée directement sur les échantillons, sur des surfaces de $5 \mu \mathrm{m}$ de diamètre.

Sur la tête féminine, plusieurs niveaux de polychromie sont nettement identifiables. La préparation la plus profonde est à base de calcite avec un peu de terre potassique magnésienne; les carnations actuellement très fines et lacunaires (sur le front au-dessus de l'œil droit) sont rosées et obtenues par un mélange de blanc de plomb et de vermillon (fig. 12). Des reprises sont ensuite visibles: une au niveau des carnations (côté droit près de l'œil sur un reste de carnations anciennes), mêlant calcite, terre et pigments au plomb, et l'autre sur le foulard. Cette dernière repose sur un enduit épais de sulfate de calcium et la teinte orangée du foulard est due à l'emploi de minium (fig. 13 et 14).

Les quatre échantillons réalisés sur la tête masculine révèlent une stratigraphie homogène: une préparation à base de calcite renfermant un peu de terre (notamment dans le chantus interne de l'œil gauche et l'oreille droite) et une carnation rosée, réalisée avec un mélange de blanc de plomb et de vermillon. La couleur des cheveux est obtenue par l'emploi de terre potassique renfermant du manganèse, ce qui est appelé terre d'ombre.

Malgré l'absence de prélèvements comportant toutes les couches, les observations effectuées sur les chevelures des deux têtes ont validé l'hypothèse d'une succession de deux traitements. Sur la tête masculine, sous la couche dominante, une couche plus orangée correspond à une première polychromie (fig. 10). Les deux niveaux sont séparés par une
Fig. 11. Tête

féminine, détail des cheveux brun sombre sous la couche plus rouge. (C) $\mathrm{C} 2 \mathrm{RMF}$ / Sandrine PagèsCamagna.
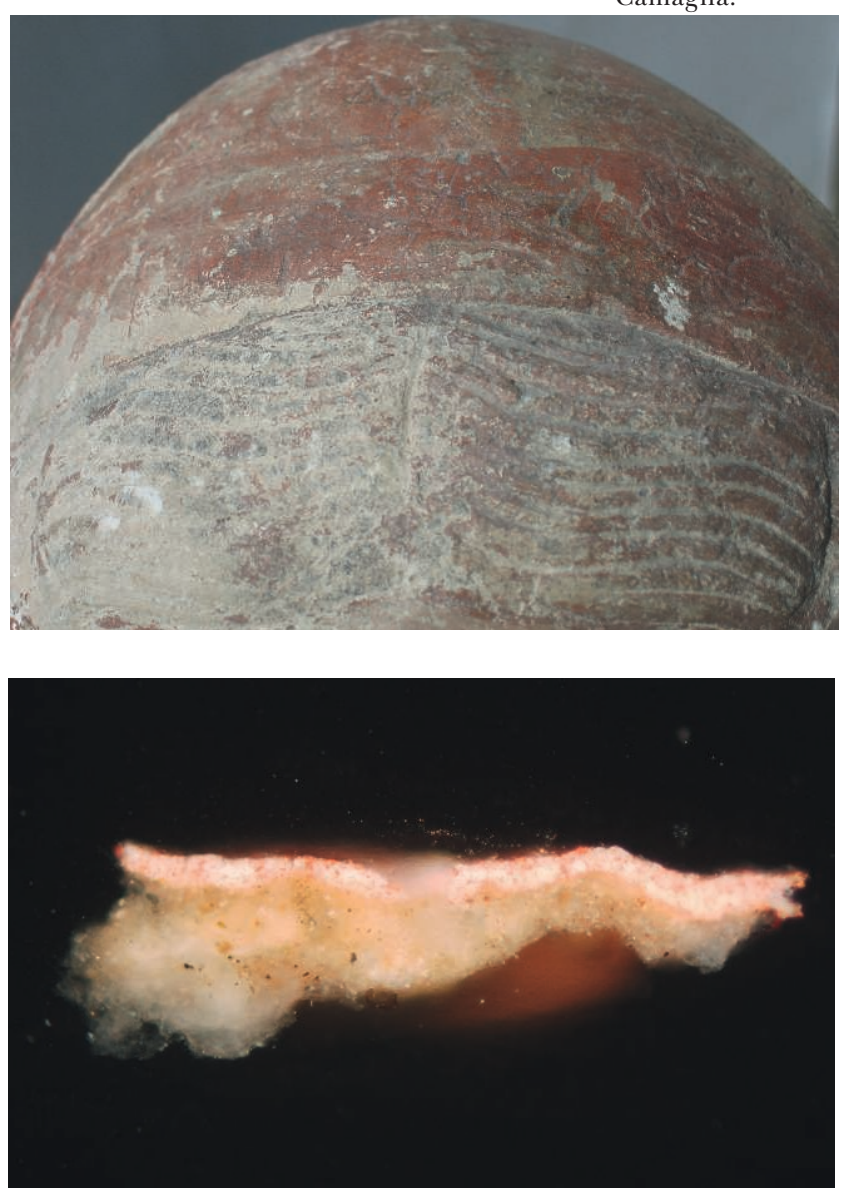

Fig. 12. Carnation de la figure féminine: la coupe stratigraphique révèle une préparation à base de calcite et de terre magnésienne, avec des carnations mêlant blanc de plomb et vermillon.

2-couche rosée: blanc de plomb avec du vermillon

1-couche beige: carbonate de calcium avec un peu de terre potassique magnésienne.

(c) C2RMF/Sandrine Pagès-Camagna.

préparation blanche. Sur la tête féminine, une couche initiale noire (à base de noir de combustion) a été détectée sous la couche brun-rouge dominante (riche en hématite); une préparation blanche intermédiaire a également été observée (fig. 11). La chevelure du personnage masculin, dans son état d'origine, était donc orange-brun ou rousse, tandis que la chevelure du personnage féminin était noire.

Dans l'œil droit du portrait féminin, le tracé de la pupille grise (mélange de blanc et de noir, sans ajout de bleu) correspondant à la première polychromie a été décelé. La pupille est légèrement plus petite que celle appartenant au traitement pictural secondaire.

Des points communs entre les polychromies des deux têtes apparaissent clairement: les carnations ont une composition identique (blanc de plomb et vermillon) et reposent 


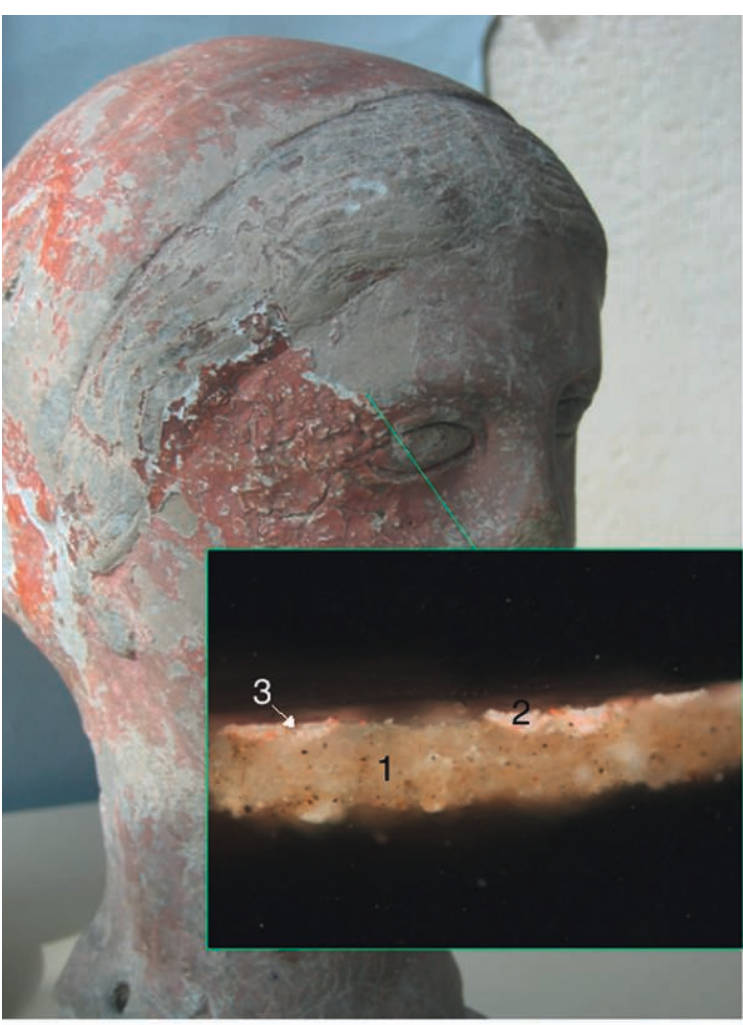

sur le même type de préparation (calcite avec un peu de terre) (fig. 12) ; de plus, le traitement pictural des yeux est comparable, avec l'utilisation de blanc bleuté pour colorer le fond de l'œil. Ces similitudes, tant dans la technique que dans le choix des matériaux, peuvent indiquer que les deux sculptures ont été repeintes à la même période, peut-être dans le même atelier. Les reprises postérieures, comme la restauration de la partie droite de la tête féminine (fig. 13), se distinguent par des compositions différentes de la préparation et des couches colorées renfermant des pigments au plomb comme le minium, dans les carnations comme sur le foulard (fig. 14).
Fig. 13. Stratigraphie de la carnation de la figure féminine avec la reprise à base de pigments au plomb (minium et blanc de plomb), sur la carnation plus ancienne contenant du vermillon. La préparation est à base de calcite et de terre magnésienne.

3-couche rosée: carbonate de calcium avec un peu de terre et de pigments au plomb (minium et/ou blanc de plomb).

2-couche rosée: blanc de plomb avec du vermillon. 1-couche beige: carbonate de calcium avec un peu de terre potassique magnésienne.

(c) C2RMF/Sandrine Pagès-Camagna.

Ces œuvres constituent donc un exemple rare conservé de restauration ancienne, effectuée à la période romaine ou plus tardivement, après le IV ${ }^{\mathrm{e}}$ siècle ou à la période médiévale, étant donné la nature des pigments utilisés, pour les carnations notamment, et dont l'usage remonte à l'Antiquité.

\section{Conclusion}

Des événements similaires semblent jalonner l'existence de ces deux têtes. Six périodes ou séquences plus ou moins longues peuvent être proposées, à partir de l'étude de leurs altérations survenues depuis leur fabrication et des investigations scientifiques conduites sur leurs polychromies:

- une première utilisation en tant qu'élément d'un monument funéraire ou d'une statue composite (contexte religieux ou profane?) à la période romaine, dans les premières décennies de l'Empire;

- la fin de cette utilisation marquée par une dissociation de la sculpture de son support (présence de coups de ciseaux autour d'une des deux mortaises, certaine pour la tête féminine, hypothétique pour la tête masculine). À ce moment, le portrait féminin semble avoir subi des dégradations structurelles majeures;

- un séjour, probablement de longue durée, en extérieur (érosion des surfaces), dans un environnement végétal (présence d'empreintes de fines racines sur les surfaces des deux

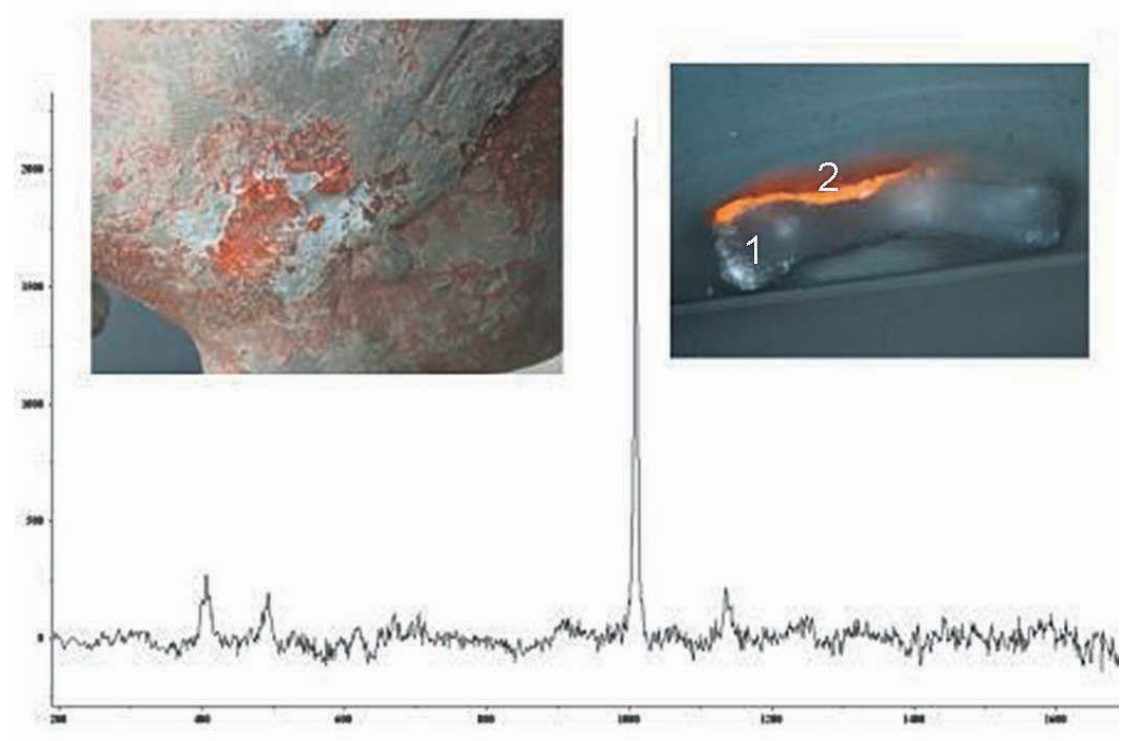

Fig. 14. Reprise du

foulard de la statue

féminine: coupe

stratigraphique révélant

du minium en surface et

du gypse en préparation,

et le spectre Raman de

la préparation.

2-couche orangée

(minium).

1-couche blanche

(calcite avec des cristaux

aciculaires de gypse).

(c) C2RMF/Sandrine

Pagès-Camagna. 
têtes, altérations antérieures au traitement pictural secondaire) ;

-une redécouverte des deux ouvres et leur restauration pour une réutilisation dans un contexte religieux ou plus probablement profane, à la période romaine ou, plus tardivement, après le $\mathrm{IV}^{\mathrm{e}}$ siècle ou à la période médiévale. L'arrière de la tête masculine est affranchi, peut-être pour une présentation frontale, et la sculpture est entièrement repeinte. La tête féminine est restaurée (bouchages des dégradations structurelles), et sa polychromie est aussi entièrement reprise;

-l'abandon ultime des deux têtes sans doute lié à celui de leur deuxième contexte d'utilisation (enfouissement des témoignages matériels) ;

- les circonstances de la deuxième redécouverte des œuvres, survenue probablement au XIX ${ }^{\mathrm{e}}$ siècle, restent inconnues (collecte par un marchand d'antiquités qui l'a ensuite cédé à Guillaume-Rey? Découverte fortuite par lui-même lors de ses excursions archéologiques en compagnie d'Eugène Grasset?).

Ainsi, il apparaît que la restauration ancienne des deux têtes pour leur réutilisation - et cela en dépit de dégradations structurelles majeures en ce qui concerne la représentation féminine - est un fait peut-être significatif de la rareté des sculptures en ronde-bosse dans le contexte de leur première redécouverte. Toutefois, l'intention d'un tel traitement nous échappe: on peut supposer la perpétuation de la tradition des portraits d'ancêtres en contexte domestique (on serait alors certainement très tôt dans la période du Bas-Empire). Ou l'intégration d'éléments de sculpture d'agrément dans un cadre architectural (démarche qui aurait pu avoir lieu aussi bien à la fin de l'Antiquité qu'au Moyen Âge ou à la Renaissance). Quoi qu'il en soit, leur caractère exceptionnel peut assurément être reconnu aujourd'hui.

\section{Notes}

1. Tête masculine: inv. N 1477 ; dimensions: H. 29; 1. 16,5; ép. 15,5 cm. Tête féminine: inv. N 1086; dimensions: H. 25,5; 1. 13,5 ; ép. $21,5 \mathrm{~cm}$

2. Ce fonds a fait l'objet d'une étude approfondie publiée par Annie Caubet (Caubet, 1984) ; nous reprenons ici l'essentiel des éléments documentaires relatifs à cette collection et collectés alors par Aleth Echalier et Marielle Pic.

3. Guillaume-Rey, [s.d.] et 1866.

4. Léon Heuzey saluait la clairvoyance de Guillaume-Rey qui avait «donné au Louvre plusieurs séries de figurines chypriotes à une époque où cette classe de monuments était encore presque inconnue dans les musées de l'Europe» (Heuzey, 1891).

5. Pour un résumé des données stylistiques sur les séries de têtes votives, voir Bonora-Andujar, 2012.

6. Jouxtant la cour Visconti, ces salles ont ouvert au public en septembre 2012, sous le nom «Orient méditerranéen dans l'Empire romain ». Elles présentent un millier d'objets provenant des trois départements antiques du Louvre (Antiquités égyptiennes, Antiquités orientales, Antiquités grecques, étrusques et romaines)

7. A. Caubet, 1984, p. 226, nº 24.

A. Hermary, 1989, p. 384.

8. Center For Archaeological Sciences, The University of Georgia (Athens, Georgia), 1987. Sans titre (analyses isotopiques d'échantillons prélevés sur dix œuvres de la Collection chypriote du musée du Louvre). Rapport adressé à madame Annie Caubet, daté du 23 novembre 1987. Document conservé au département des Antiquités orientales.

9. A. Caubet, 1984 , p. $226, \mathrm{n}^{\circ} 25$.

10. Y. Coquinot, 2010.

11. Ch. Pariselle, 2010

12. S. Pagès-Camagna, 2009.

13. Localisation des prélèvements sur la tête masculine: oreille droite, base des cheveux au niveau de la nuque, chevelure derrière l'oreille gauche et chantus interne rouge de l'œil gauche. Sur la tête féminine foulard orange dextre (partie usée et reprise), carnation au-dessus de l'œil droit (partie usée), carnation originale et première reprise au-dessus de l'œil droit, cheveux bruns (côté gauche de la raie médiane) et roses derrière la boucle d'oreille gauche.

\section{Bibliographie}

Bonora-Andujar I., 2012, "Les têtes votives à Chypre: une longue tradition" dans Bel N. et al., L'Orient romain et byzantin, musée du Louvre/éditions Actes Sud, Paris, p. 192-194, Fig. 177.

Calligaro T, Dran J.-C., Salomon J., 2005, "Ion beam microanalysis, in Nondestructive microanalysis of cultural heritage materials", dans Comprehensive Analytical Chemistry XLII (eds. K. Janssens and R. Van Grieken), Elsevier Amsterdam, p. 227-276.

Caubet A., 1984, «Aux origines de la collection chypriote du Louvre: le fonds Guillaume-Rey (1860-1865) », Report of the Department of Antiquities Cyprus, 1984, p. 221-229, notamment p. $226 \mathrm{n}^{\circ} 24$ et $^{\circ} 25, \mathrm{Pl}$. XLV: 4, 5, 8, 9

Center For Archaeological Sciences, The University of Georgia (Athens, Georgia), 1987. Sans titre (analyses isotopiques d'échantillons prélevés sur dix ouvres de la Collection chypriote du musée du Louvre). Rapport adressé à Madame Annie Caubet, daté du 23 novembre 1987.

Coquinot Y., 2010, Tête féminine sculptée polychrome, Chypre (Époque romaine impériale), Inv. N 1086. Tête masculine sculptée polychrome, Chypre (Époque romaine impériale), Inv. $N$ 1477. Louvre$D A O$ - Examen et analyse de la pierre Rapport d'étude C2RMF 17186B, 18 octobre 2010, Centre de Recherche et de Restauration des Musées de France - C2RMF, Laboratoire de recherche des musées de France, Département Recherche.

Eaton, S. et Robertson, A., 1993, "The Miocene Pakhna Formation, southern Cyprus and its relationship to the Neogene tectonic evolution of the Eastern Mediterranean. Sedimentary Geology", 86, p. 273-296.

Folk, R. L., 1959, "Practical petrographic classification of limestone's", American Association of Petroleum Geologists Bulletin, 43 , p. $1-38$.

Guillaume-Rey E., [s.d.], Voyage dans le Haouran et aux abords de la Mer Morte exécuté pendant les années 1857 et 1858, Paris.

Guillaume-Rey E., 1866, «Rapport sur une mission scientifique accomplie en 18641865 dans le N. de la Syrie », Archives des Missions scientifiques, $2^{\mathrm{e}}$ série 3II, p. 329373.

Hermary A., 1989, Catalogue des antiquités de Chypre. Sculptures. Musée du Louvre, département des Antiquités orientales, Paris, p. 67, n 102; p. 384, n 784.

Heuzey L., 1891, Musée national du Louvre, Catalogue des Figurines Antiques de Terre Cuite, Paris

Pagès-Camagna S., 2009, Tête masculine et tête féminine, Pierre polychromée, Chypre, époque romaine, $I^{e r}$ siècle après J.- $C$. Rapport d'étude C2RMF 17186, 6 avril 2009, Centre de Recherche et de Restauration des Musées de France C2RMF, Laboratoire de recherche des musées de France, département Recherche

Pariselle C., 2008, Études préliminaires pour la séance du 17 avril 2008, mai 2008. Portrait d'un personnage masculin. Portrait d'un personnage féminin.

Pariselle C., 2010 (1 et 2), Rapports d'étude et de restauration, mars 2010 . (2 vol.). Tête masculine, Sculpture (Pierre) N 1477. Tête féminine, Sculpture (Pierre), N 1086.

Pariselle C., 2010 (3), Bilan de l'étude des têtes sculptées et polychromées, N 1477 et N 1086 (Chypre), novembre 2010 